\title{
General practitioners' perceptions, attitudes and experiences of frailty and frailty screening
}

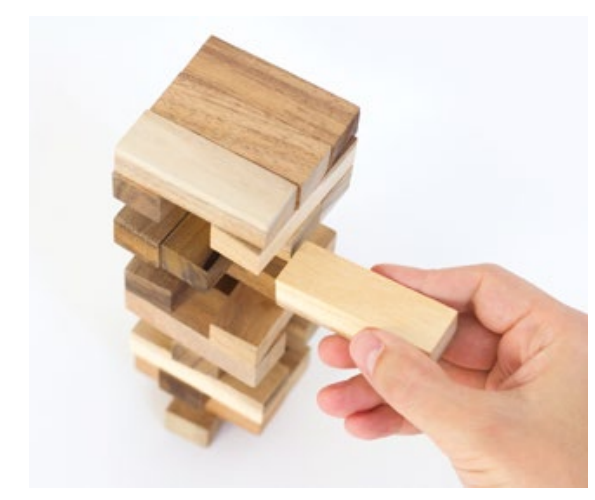

CPD

Rachel C Ambagtsheer, Mandy M Archibald, Michael Lawless, David Mills, Solomon Yu, Justin J Beilby

\section{Background and objectives}

General practitioners (GPs) are uniquely positioned to support frailty identification and management. However, awareness of frailty and its treatment remains an emergent concept for many. Consequently, our aim was to explore GPs' perceptions, attitudes and experiences of frailty and frailty screening.

\section{Methods}

A qualitative focus group study was conducted with 22 South Australian GPs. GPs were recruited through a combination of purposive, convenience and snowball sampling. Data were analysed using a thematic analysis approach.

\section{Results}

GPs saw frailty as a cycle of worsening decline punctuated by experience of negative outcomes. Participants largely felt that they already knew who their frail patients were without the need for formal screening. Consequently, there was varied support for formal screening, largely dependent on its intended purpose. Few GPs had actively intervened to prevent the onset or progression of frailty, with most strategies aimed at stabilisation and management.

\section{Discussion}

This study suggests that Australian GPs may be open to a proactive approach to frailty assessment and treatment, given appropriate training and resources.
FRAILTY is a clinical syndrome heightening an individual's vulnerability to risk of adverse health outcomes when exposed to stressors. ${ }^{1,2}$ It is highly prevalent among older people ${ }^{3}$ and associated with many negative health outcomes, including increased risk of premature death, falls, hospitalisation, dependence and physical limitation. ${ }^{4}$ Despite these negative consequences, frailty is often under-treated by health service providers, especially within the context of single diseasefocused, non-person centric and fragmented healthcare systems. ${ }^{5,6}$ This shortcoming is especially pertinent given evidence suggests that frailty is treatable through appropriate and well-timed intervention. ${ }^{7-11}$ While the aetiology of frailty is not completely established, there is increasing understanding of the contribution that the clinical manifestations of frailty make to the worsening of the frailty cycle; for example, how undernutrition may lead to increasing loss of muscle mass, and thus to declines in walking speed, increased exhaustion, and so on. ${ }^{12}$ The emerging evidence suggests that some of these causal factors may be reversible, ${ }^{13}$ leading to the reversal of frailty status itself, ${ }^{14}$ and thus that proactive identification of frailty and its components offers an opportunity to delay or avert negative outcomes. ${ }^{15,16}$

Screening for frailty has been promoted as one means to address widespread under-recognition of frail older adults within primary care. ${ }^{1}$ Historically, health system responses to frailty have tended to be reactive and have occurred at the acute end of the spectrum. ${ }^{6}$ Given a global emphasis on reorienting the health system towards integrated and person-centred care for older people, ${ }^{17-19}$ some have argued that general practitioners (GPs) should play a central part in frailty identification and management. ${ }^{20,21}$ Within the $\mathrm{UK}$, this view has been reflected in the development of the GP contract that requires GPs to detect and treat all patients aged $\geq 65$ years with moderate-to-severe frailty. ${ }^{22,23}$ However, addressing frailty within primary care settings has been labelled both 'an emerging concept' ${ }^{5}$ and 'a topic in its infancy' ${ }^{24}$ Despite increasing attention being given to the concept of frailty within the past decade, ${ }^{25,26}$ only a small number of studies have specifically addressed 
GP perceptions and attitudes towards frailty, ${ }^{27,28}$ and to our knowledge none have explored attitudes to screening outside of the context of specific interventions. ${ }^{29}$ Consequently, many unanswered questions remain.

In this study, we aimed to explore the perceptions, experiences and attitudes of South Australian GPs as a foundation to understand the context for frailty screening implementation within Australia. Our research questions were: What are GPs' perceptions, attitudes and experiences towards the concept of 1 ) frailty and 2) frailty screening?

\section{Methods}

This project is part of a broader research program exploring perspectives on frailty and frailty screening among key stakeholders within Australia. ${ }^{30} \mathrm{We}$ adopted a qualitative descriptive design using a focus group methodology. Focus groups are a 'data collection technique that capitalises on the interaction within a group to elicit rich experiential data' ${ }^{31,32}$ Within the context of this study, the dynamic nature of the focus groups allowed for the drawing out of a wider range of perspectives than would be achieved through other methods, such as individual interviews or a survey design.

We obtained ethical approval through the Torrens University Higher Research Ethics Committee (HREC Reference number: H10/17). All participants received written information and signed consent forms. Participants were reimbursed commensurately for their time.

\section{Setting}

The study was conducted in Adelaide, South Australia, between September and December 2016.

\section{Participants and recruitment}

We adopted a stratified, purposeful scheme combining purposive, convenience and snowball sampling to recruit GPs, seeking to obtain moderately homogenous sub-groups. ${ }^{33}$ The key principle guiding our sampling was to provide a baseline exploration of Australian GPs' perspectives and to illustrate a range of views on the topic. Sub-group stratification was intended to generate theoretically meaningful comparative detail, with urban/rural location and age being key considerations. We based this decision on widely observed disparities between Australian urban and rural areas, especially with respect to service accessibility ${ }^{34-36}$ and health status ${ }^{36,37}$ and to prior research showing that GPs' age may have a strong influence on clinical practice style, work practices and quality of care. ${ }^{38-40}$

Consequently, we deliberately sought to recruit GPs to participate in at least three focus groups comprising between six and nine participants in each: ${ }^{30}$ one urban 'older group' (focus group [FG] 1; GPs aged 40 years and over); one urban 'younger group' (FG2; GPs aged under 40 years); and one rural group (FG3; GPs of any age). A local senior GP led the recruitment effort; we additionally requested that recruited participants invite their contacts to join the groups. In a number of cases, we also contacted practices directly to request expressions of interest.

\section{Data collection}

We developed a semi-structured interview guide on the basis of our research questions (Appendix 1). A practising GP reviewed and provided feedback on the topic guide. An experienced academic GP (JB) conducted the focus groups with support from members of the research team. Focus groups were audio-recorded using a password-encrypted mobile device and securely video-recorded using the Zoom software platform, with the exception of the rural focus group, which was both audio-recorded and video-recorded using Zoom. The mean duration of each focus group was 101 minutes (range: 76-123 minutes). All sessions were transcribed verbatim by a professional transcription service.

\section{Analysis}

Data analysis progressed through several stages as the research group worked to identify an analytic process appropriate for the complex nature of the focus group data. In the first stage, RA conducted an inductive line-by-line coding of transcripts within NVivo (v.11 Pro). Codes were generated on the basis of several readings of the transcripts. These codes and a number of preliminary emergent themes were discussed in consultation with a second researcher (MA). However, because of a shared sense that the coding was taking place at too granular a level to appropriately address the research questions, a second round of coding and analysis was determined.

In the second phase, a framework analysis (FA) approach ${ }^{41}$ was applied within NVivo and Microsoft Excel. FA is an approach suited to research with specific questions, limited time frames, a particular sample population and a priori issues for exploration. ${ }^{42,43}$ One analyst (RA) developed a preliminary coding framework on the basis of her familiarity with the data. This framework was subsequently refined by a team of experienced qualitative researchers and a second member of the research team (PDM), after which the final category refinements were made. Segments of text were coded to the framework categories and charted for each participant within the framework matrix. Two research team members (RA and ML) subsequently analysed the summaries independently for meaning.

Lastly, a final inductive recoding of all transcripts was conducted by RA, using a combination of Microsoft Word and OneNote. Although the initial two phases of analysis informed the overall approach, and the emergent codes at each stage were compared, the final themes were developed during this phase. Throughout the process, two research team members (MA and ML) were consulted to discuss conceptualisation of the emerging themes, with RA making the final decisions on theme and sub-theme formulation.

\section{Results}

Demographic characteristics of participants are shown in Table 1. Broad themes identified were 'frailty as a worsening cycle of decline', 'rapid, intuitive screening based on visual cues', 'support for formal screening depends on its purpose and context' and 'frailty action 
as stabilisation rather than improvement'. These themes are summarised in Table 2, along with corresponding sub-themes.

\section{Frailty as a worsening cycle of decline} Participants generally understood frailty as a worsening cycle of decline over time, characterised by a series of negative outcomes. Two variations on the 'cycle of decline' concept were described, with one reflecting a 'past' orientation of advanced decline, and the other a 'future' orientation of prospective risk. The degree to which each perspective was held by participants tended to vary systematically across focus groups, with the 'Older urban' group almost exclusively holding the first perspective and the 'Younger urban' group almost exclusively the latter. The 'Rural' group held mixed views across the two perspectives, although views held tended to align similarly with the age cohort of individual GPs.

The first view conceptualised frailty as an increased risk of negative outcomes, in which participants described patients as having a significantly elevated risk for negative outcomes including hospitalisation, falls, fractures, institutionalisation, illness, disease and malnutrition. Similarly, the consequences of adverse health events were also perceived as elevated because of patients' frailty status. Some described the outcomes as having a more intensive effect than usual because of the patient's increased vulnerability, as one GP noted:

[It's] more of a vulnerable kind of state ... that ... leads to greater consequence in terms of illness and disease. (Female GP, FG2)

The second conceptualisation of frailty discussed was of frailty as a progressive loss of functional ability, suggestive of a state of increasing disability. Participants described a recognisable pattern characterised by diminishing function that typically leads to declining independence, increasing limitation in activities of daily living and, ultimately, reduced quality of life. As described by one GP:

Somebody becomes frail when they don't do what they used to be able to do, at the same time, in the same fashion, in the same activity... (Male GP, FG1)

Regardless of their orientations towards either position, participants generally described this worsening progression of frailty as initiated by a major trigger event, representing a view of frail patients as crossing some type of threshold. This was likened by one GP to falling off a tightrope:

A lot of them ... to me seem like they are walking a tightrope ... only something small has to happen, and ... they'll become frail old people. (Male GP, FG1)

Trigger events identified by participants were commonly of two types: a significant health event such as a fall, fracture, illness (eg pneumonia), diagnosis of disease (eg ischaemic myopathy, neuropathy) or hospitalisation; or a significant life change, such as grief/loss (eg widowhood), retirement or loss of licence.

According to participants, trigger events were followed by further compounding events or factors considered to worsen frailty. As one GP stated:

I think it's probably a bit self-perpetuating as well ... it ... can start with one major issue, and then it just seems like everything on top of that, it takes so much less to bring on ... (Female GP, FG2).

In particular, GPs agreed that hospitalisation was a critical period during which a patient's condition might worsen, in part because of reduced mobility. No participants described frailty as being multidirectional (ie can both improve and worsen) or reversible unless prompted by the interviewer.

GPs frequently emphasised the 'multifactorial' nature of frailty. As one participant said:

\section{I think of it as a combination of multiple components... many factors that in combination make a person frail, rather than just one or two of them. (Female GP, FG2)}

This multifactorial nature of frailty was considered to apply across three key aspects of frailty: trigger events, compounding factors and impacts of frailty. For example, social isolation was seen to be an example of all three, in the sense that it could act as an initiator, a compounder and an impact of frailty.

Some GPs commented on the perceived rate of decline of their patients when discussing the progression of frailty. GPs differed with respect to how quickly they perceived the general rate of decline to be, although there was no systematic variation across focus groups. While some described frailty as 'slow gradual decline', others reported noticing 'sudden' change. GPs in FG1 acknowledged that this perception was likely related to how frequently they saw the patients, reinforcing the importance of continuity of care. For example, one GP observed that:

... it depends how frequently you see them. Ifyou're seeing them once every couple of weeks ... It might be a gradual decline, but ifyou see them - like, I might see them every two or three months, it's dramatic decline. (Male GP, FG1)

\section{Table 1. Participant characteristics} ( $n=22)$

\begin{tabular}{lcc}
\hline Characteristic & n & $\%$ \\
\hline Sex & 9 & 40.9 \\
\hline Male & 13 & 59.1 \\
\hline Female & & \\
\hline Age (years) & 12 & 54.5 \\
\hline$<35$ & 5 & 22.7 \\
\hline $35-59$ & 5 & 22.7 \\
\hline$\geq 60$ & 3 & 13.6 \\
\hline Professional experience (years) & & \\
\hline$<5$ & 9 & 40.9 \\
\hline $5-10$ & 1 & 4.6 \\
\hline $11-20$ & 9 & 40.9 \\
\hline$>20$ & 15 & 68.2 \\
\hline Location & 7 & 31.8 \\
\hline Metropolitan & & \\
\hline Non-metropolitan & & \\
\hline
\end{tabular}




\section{Rapid, intuitive screening based on visual cues}

None of the participants in the sample used formal screening instruments to detect frailty, but most regularly applied a type of rapid, intuitive screening when interacting with older patients. GPs repeatedly warranted their informal, intuitive screening practices (ie performing screening 'in their heads') by characterising frailty as 'obvious' and, often, 'visual'. This viewpoint was exemplified by one GP, who said:

I think ... you know, you can assess people's frailty within four seconds of looking at them, really... (Male GP, FG3).

There was a prevailing view among participants that they already knew who their frail patients were on the basis of frequent contact with them and, consequently, they could potentially readily choose them from a list without the need for formal tools.

GPs described relying on a number of typical warning signs - 'red flags' - as alerting them to the possibility that a patient might be frail. Examples included non-mobility related warning signs such as frequent and/or recurrent presentations to the GP, depression, hearing decline, polypharmacy, emergency room visits, weight loss and decline in hygiene/ self-care. However, for most participants, the best means of identifying frailty was simply observing patients' movements; examples included walking down a corridor, getting in and out of chairs, and getting up onto an operating table or a bed. One GP stated:

... within the first couple of seconds you know what's going on; you can see how long it takes them to get up, you can see if they use the armrest, you see if they don't need to do that, if they've got a walker or a frame or a stick, or if someone's helping them, if they're stooped over, their pace within the room. (Male GP, FG3)

However, some GPs acknowledged that recognising frailty subjectively on the basis of visible physical indicators might result in missed opportunities to address frailty earlier. The sense of missed opportunity was mentioned in relation to a greater time lag between the onset of frailty and patient presentation to the GP, where identifying frailty was dependent on the GP noticing it. As one participant stated:

... [by] the time they present, and you notice it, you wonder what else has been happening before that. Six months, 12 months before that ... (Male GP, FG1)

\section{Table 2. Participant themes and sub-themes}

\begin{tabular}{ll}
\hline Theme & Sub-theme \\
\hline $\begin{array}{l}\text { Frailty as a worsening cycle } \\
\text { of decline }\end{array}$ & Frailty as increased risk of negative outcomes \\
& Frailty as progressive loss of functional ability \\
& Trigger events and frailty threshold \\
& Compounding factors worsening frailty \\
& Frailty as multifactorial \\
\hline Rapid, intuitive screening based & Sudden versus gradual rate of decline \\
on visual cues & Frailty as obvious and visual \\
& Red flags (typical signs of frailty) \\
\hline $\begin{array}{l}\text { Support for formal screening } \\
\text { depends on its purpose and }\end{array}$ & Missed opportunities to identify frailty earlier \\
context & Universal screening as pre-emptive risk profiling \\
\hline $\begin{array}{l}\text { Frailty action as stabilisation } \\
\text { rather than improvement }\end{array}$ & Fargeted screening as case-finding \\
\hline
\end{tabular}

GPs who expressed this sentiment also expressed a sense of frustration that they may have been able to prevent the onset of frailty if appropriate intervention had been employed earlier.

\section{Support for formal screening depends on its purpose and context}

Participants varied in terms of their degree of support for the introduction of formal screening, although this variation was not systematic among the groups. While many felt that frailty screening would be (theoretically) useful in the sense of risk profiling, some were concerned that, in practice, screening might translate to a perfunctory exercise that would not be sufficiently person-centred. There was also a strong sense that GPs were already overwhelmed with the demands of a busy work environment, so that the rationale for introducing additional screening would need to be made clear to practitioners and patients. Consequently, support for screening tended to be contingent on its intended purpose and context. As a result, GPs distinguished between universal and targeted screening (case-finding), and their respective aims, as described by one GP, who stated:

One is detecting at risk ... versus one already with a condition.... That's more an assessment of how bad it is; the other one is ... a predictive value about where this may be going ... (Male GP, FG1)

The possibility of universal screening - in the sense of making screening available to everyone of a certain age - was viewed by some as potentially useful in predicting risk of frailty before its onset. Perceived advantages included the ability to avert negative outcomes, the ability to screen infrequent attendees at the practice, the ability to identify patients at risk of frailty who otherwise appeared well and the possibility of using screening results as a means of patient motivation. As one GP observed:

... ifyou say, 'Well you're at risk of negative consequences of ageing', ... it's a really good opportunity to ... kick into their purpose and what they want from life, so that it 
turns it into a really good conversation and then you can link change in their life into what they want. (Male GP, FG2)

However, there was no consensus regarding when universal screening should start, with the broad age range proposed being 50-75 years. Participants proposing the age of 75 years generally rationalised this by linking it to the $75+$ health assessment (a free annual health check), ${ }^{44}$ but others thought that this was too late.

Some GPs felt that targeted screening in the sense of case-finding for patients who were already frail might also prove useful. Targeted screening was generally viewed as a more intensive exercise that echoed the typical signs mentioned by GPs in recognising frailty. Such activity was viewed as potentially more time intensive by the GPs, and so integration with existing care plans, along with the involvement of practice nurses, was a commonly proposed solution. However, a number of GPs in FG2, many of whom had recently graduated, seemed less willing to delegate this task to nurses. As one GP said:

\section{... I think it's good to ... do it yourself so} that you know exactly what you're doing ... or else you lose track of what's actually in the plan. I'd worry about signing off on things that you won't know what happened. (Female GP, FG2)

\section{Frailty action as stabilisation rather than improvement}

In reflecting on their experiences with older patients, few GPs reported having actively intervened to prevent the onset of frailty. When questioned by the interviewer on this point, GPs described hypothetical scenarios in which they possibly could have applied measures to address frailty, but did not - that is, they spoke in terms of what could be done rather than what had been tried. Possible strategies raised by GPs to prevent frailty included resistance training, medication management, nutrition interventions and proactive referral to specialist services such as allied health services and chronic disease clinics. In this way, there was significant crossover between frailty prevention and frailty management strategies, as commented on by one GP:

A lot of our answers are more about how we manage frailty when it's obviously in front of us ... and so I don't really know how to prevent frailty ... (Female GP, FG3)

GPs spoke more readily about their experiences of frailty management. For most GPs, frailty management was focused on stabilising the patient at their current point of frailty progression to maximise their independence and avoid further decline. Strategies reflected a type of 'GP as gateway' (and, by extension, 'practice as gateway') model, wherein the GP and their team worked to identify key patient issues and then refer to other services accordingly. Management strategies raised by GPs centred on modifying key patient lifestyle behaviours to improve aspects of the patient's condition. These included encouraging physical activity, resistance training and healthy nutrition; addressing falls and balance; and managing medications. Strategies addressing social isolation were seen as particularly useful. Key services identified for referral included exercise physiologists, physiotherapists, Falls and Balance Clinics and community programs. Referral was commonly presented as a practical solution to the problem of personally motivating patients or supervising a program of activity, which some GPs said that they did not have the time or skill set to do, as one GP noted:

I encourage a lot of people to go to the gym but they just ... they just don't do it. They ... find it too boring or they just can't be bothered. So, I think having someone supervise a program for them is probably more critical; it's not something as GPs we've got time to do. (Male GP, FG3)

Patient motivation was frequently identified by GPs as being a key determinant of whether frailty intervention (and also frailty screening) was likely to be successful or not. Participants often portrayed their discussions with frail older patients about issues relating to frailty and ageing as challenging because of high levels of patient resistance. As one GP commented:

I think we all feel the sense that it's very difficult to motivate people once they're already down the slippery route.' (Female GP, FG2)

GPs gave examples of patients refusing to discuss treatment options, opting not to pursue recommended referrals and refusing to take up the $75+$ health assessment. Many participants perceived patients as having their own agendas when they came to the practice, with any deviation from this agenda viewed as an unwelcome intrusion by the patient. To counter this resistance, some GPs discussed invoking 'scare tactics' (eg risk of losing driver's licence) as a strategy where motivation proved difficult, but there was an acknowledgement that this did not work for all patients. However, these issues were largely discussed in relation to patients who are already frail. Some GPs felt that people in the younger age groups (those now in their 50s or $60 \mathrm{~s}$, and even younger) might be more receptive to the idea of screening, as reflected by this exchange between two male GPs in FG1:

... in 40 years' time, they'll probably do all this self-screening at home and say present ... with 'I think I'm, you know... at risk for this', or something...

That's what they do now, the younger ones. They come in with their print-out.

\section{Discussion}

\section{Summary of findings}

The aim of this study was to explore the perceptions, attitudes and experiences of Australian GPs towards the concept of frailty and frailty screening. GPs expressed a conceptualisation of frailty as a cycle of worsening decline punctuated by experience of negative outcomes, and characterised either by a view of frailty as a state of heightened risk or as increasing functional decline. Participants generally felt that they already knew 
who their frail patients were, describing an informal type of screening practice based on the presence of typical warning signs, although some GPs acknowledged that this may mean they are missing opportunities to act earlier. Consequently, there was varied support for screening, largely dependent on its intended purpose. Universal screening, while seen as potentially useful by some GPs, was regarded as resource-intensive. Targeted screening, akin to case-finding, was viewed as more practically incorporated within existing practice workflows. Few GPs reported having actively intervened to prevent the onset or progression of frailty, with most strategies described aimed at stabilising the patient and maintaining their independence for as long as possible. Frailty management was widely practised and largely consisted of referral to other services, with patient motivation seen as a key determinant of the ultimate outcome.

\section{Strengths and limitations}

This study is among the first we are aware of to explore the issue of GP perceptions, attitudes and experiences towards frailty and frailty screening, and the first in the Australian context. As such, the findings provide useful contextual information for current policy efforts towards improving frailty identification and management within primary care. In addition, the deliberate purposive sampling strategy allowed for a range of ages and professional experience to be represented, potentially revealing important generational differences in GP attitudes. However, there were also several limitations. The recruitment strategy meant that there was a higher degree of acquaintance between group members than may have been obtained through random sampling, signifying that the study may have attracted GPs who shared similar underlying values and work practices. Secondly, the presence of senior academic GPs either as members of the research team or as participants may have led to a degree of social desirability bias among others. Lastly, there was no representation among older female GPs within rural areas because of recruitment difficulties.

\section{Comparison with existing literature}

GPs' conceptualisation of frailty in this study accorded very closely with results from a previous survey of Canadian healthcare professionals, ${ }^{45,46}$ inclusive of the distinction between a risk orientation versus a disability orientation towards frailty. Half of the respondents $(n=356)$ in the Canadian study indicated that they felt frailty incorporated some aspect of functional loss akin to disability. ${ }^{45}$ Similarly, a recent study by Korenvain and colleagues ${ }^{28}$ indicated that GPs commonly reference functional considerations in developing their clinical impressions of frail older patients. Furthermore, there is some evidence that consumers also tend to equate frailty with disability. ${ }^{47}$ This is despite a current general consensus held by many frailty experts that frailty is a concept distinct from disability, ${ }^{48}$ although causally related, ${ }^{13,48,49}$ and perhaps reflects that contrasting opinions on this matter still proliferate. ${ }^{46}$ The fact that the GPs holding this view frequently saw patients in the advanced stages of frailty lends support for a notion of frailty as a kind of 'early stage of disability' as asserted by some, ${ }^{50}$ rather than as a separate phenomenon altogether.

The 'cycle of decline' view of frailty suggests an assumption among GPs of frailty as unidirectional rather than reversible, despite emerging evidence from the field indicating that reversibility is achievable. ${ }^{7-11}$ Such a perspective reflects previous research finding a knowledge gap in general clinician awareness of frailty ${ }^{51}$ along with similar findings among consumers. ${ }^{47}$ Although a recent meta-synthesis found that some healthcare providers do see frailty as malleable in its earlier stages, there was also an expressed belief that this malleability stopped at a certain point, beyond which it would not be useful to intervene. ${ }^{52}$ In some respects, this belief may explain why GPs in our study were mostly attempting to manage and stabilise frailty progression, rather than to reverse it.

Many GPs in our study felt that they already knew which of their patients were frail without the need for formal tests, reflecting the results of a previous study suggesting that in the absence of appropriate screening tools, GPs commonly employ a type of 'clinical gestalt' technique, or intuition, to identify frail patients. ${ }^{28}$ Further, the description of frailty as being obvious and highly visible suggests a possibility that this recognition is occurring at a relatively advanced stage of frailty progression. This issue is important because it may act as a potential barrier in addressing frailty more effectively within primary care. ${ }^{22}$ In addition, the ability of GPs to 'eyeball' frailty effectively has not been well established. Few studies have explicitly evaluated GP impression of frailty against a reference standard, ${ }^{21,53,54}$ and the supporting evidence for the validity of this approach in identifying frailty is inconclusive. Although a number of studies have reported moderate-tohigh levels of GP accuracy in identifying frailty, ${ }^{20,50,53,54}$ there are significant design limitations associated with each that have implications for the generalisability of results, including pre-training of GPs, inclusion of screening test elements and/ or unusually high frailty prevalence.

\section{Implications for general practice}

Primary care settings are ideally suited to identify and manage frailty. ${ }^{53}$ In the $\mathrm{UK}$, this position has been translated into policy in terms of the GP contract on frailty. ${ }^{23}$ However, our study suggests that more work is needed before proactive, person-centred frailty identification and management becomes an everyday reality. There is great potential for the concept of frailty to act as an organising pivot around which person-centred care of older people could operate, ${ }^{22,46,55}$ but this shift cannot - and should not - be undertaken by GPs in isolation. Our study shows that GPs are open to thinking about and addressing frailty in a different way, but that they will need to be supported with the right teams, tools, processes and systems (including policy and funding support) to enact change effectively. More effort needs to be devoted towards GP research and training on frailty, both at the medical curriculum level and as part of ongoing GP professional education. Further, greater 
public and provider awareness about frailty will likely be critical in advancing frailty prevention and treatment on a global scale. ${ }^{56}$

\section{Authors}

Rachel C Ambagtsheer BA (Hons), MlnfSt, Research Fellow and PhD Candidate, Torrens University Australia, Adelaide, SA; NHMRC CRE for TransDisciplinary Frailty Research to Achieve Healthy Ageing, Adelaide, SA. rambagtsheer@laureate.net.au Mandy M Archibald BScN, PhD, CIHR Research Fellow, NHMRC Postdoctoral Research Officer, Flinders University, Adelaide, SA; NHMRC CRE for Trans-Disciplinary Frailty Research to Achieve Healthy Ageing, Adelaide, SA

Michael Lawless BPsych (Hons), PhD, Postdoctoral Research Fellow, Flinders University, Adelaide, SA; NHMRC CRE for Trans-Disciplinary Frailty Research to Achieve Healthy Ageing, Adelaide, SA

David Mills MD, FRACGP, Adjunct Associate Professor, Adelaide Rural Clinical School, University of Adelaide, SA

Solomon Yu PhD, FRACP, MBBS, Clinical Deputy Director, Aged and Extended Care Services, Central Adelaide Local Health Network and Clinical Associate Professor, Adelaide Geriatrics Training and Research with Aged Care (GTRAC) Centre, Adelaide Medical School, University of Adelaide, SA; NHMRC CRE for Trans-Disciplinary Frailty Research to Achieve Healthy Ageing, Adelaide, SA

Justin J Beilby MBBS, FRACGP, MD, MPH, DA DRCOG, Vice-Chancellor, Torrens University Australia, Adelaide, SA; NHMRC CRE for TransDisciplinary Frailty Research to Achieve Healthy Ageing, Adelaide, SA

Competing interests: None.

\section{Appendix 1. Interview guide}

- What is your understanding of the concept of frailty? How would you define it from a clinical perspective?

- When would you say that someone is frail?

- What is your understanding of how frailty develops? How does it progress?

- What, if anything, can be done to prevent people from becoming frail? What can be done once a person is frail?

- What can general practitioners (and other healthcare professionals) do to improve care of pre-frail and frail persons?

- Can you describe your attitude towards the concept of frailty screening? Under what circumstances, if any, do you feel it would be useful?

- At which time points and in what settings would it be most useful?

- What would make a screening tool most feasible for use within the context of a standard general practice consult?
Funding: This study was funded by grants from the National Health and Medical Research Council and Resthaven/Geriatrics Training \& Research with Aged Care (G-TRAC).

Provenance and peer review: Not commissioned, externally peer reviewed.

\section{References}

1. Morley JE, Vellas B, Abellan van Kan G, et al. Frailty consensus: A call to action. J Am Med Dir Assoc 2013;14(6):392-97. doi: 10.1016/j. jamda.2013.03.022

2. Walston J, Hadley EC, Ferrucci L, et al. Research agenda for frailty in older adults: Toward a better understanding of physiology and etiology: Summary from the American geriatrics society/ national institute on aging research conference on frailty in older adults. J Am Geriatr Soc 2006:54(6):991-1001. doi: 10.1111/j.15325415.2006.00745.x

3. Collard RM, Boter $H$, Schoevers RA Oude Voshaar RC. Prevalence of frailty in community-dwelling older persons: A systematic review. J Am Geriatr Soc 2012;60(8):1487-92. doi: 10.1111/j.1532-5415.2012.04054.x.

4. Vermeiren S, Vella-Azzopardi R, Beckwée D, et al. Frailty and the prediction of negative health outcomes: A meta-analysis. J Am Med Dir Assoc 2016;17(12):1163.e1-1163.e17. doi: 10.1016/j. jamda.2016.09.010.

5. De Lepeleire J, Iliffe S, Mann E, Degryse JM. Frailty: An emerging concept for genera practice. Br J Gen Pract 2009;59(562):e177-82. doi: 10.3399/bjgp09X420653.

6. Turner G, Clegg A. Best practice guidelines for the management of frailty: A British Geriatrics Society, Age UK and Royal College of General Practitioners report. Age Ageing. 2014;43(6):744-47. doi: 10.1093/ageing/afu138.

7. Cameron ID, Fairhall N, Langron $\mathrm{C}$, et al. A multifactorial interdisciplinary intervention reduces frailty in older people: Randomized trial. BMC Med 2013;11(1):65. doi: 10.1186/1741-701511-65.

8. Kim H, Suzuki T, Kim M, et al. Effects of exercise and milk fat globule membrane (MFGM) supplementation on body composition, physical function, and hematological parameters in community-dwelling frail Japanese women: A randomized double blind, placebo-controlled, follow-up trial. PLoS One 2015;10(2): e0116256. doi: 10.1371/journal.pone.0116256.

9. Li CM, Chen CY, Li CY, Wang WD, Wu SC. The effectiveness of a comprehensive geriatric assessment intervention program for frailty in community-dwelling older people: A randomized, controlled trial. Arch Gerontol Geriatr 2010;50S39-42.

10. Ng TP, Ling LHA, Feng $L$, et al. Cognitive effects of multi-domain interventions among prefrail and frail community-living older persons: Randomized controlled trial. Journals Gerontol Ser A 2017;73(6):806-12. doi: 10.1093/gerona/glx207.

11. Tarazona-Santabalbina FJ, Gómez-Cabrera MC, Pérez-Ros $P$, et al. A multicomponent exercise intervention that reverses frailty and improves cognition, emotion, and social networking in the community-dwelling frail elderly: A randomized clinical trial. J Am Med Dir Assoc 2016;17(5):426-33. doi: 10.1016/j.jamda.2016.01.019.

12. Fried LP, Tangen CM, Walston J, et al. Frailty in older adults: Evidence for a phenotype. J Gerontol A Biol Sci Med Sci 2001;56(3):M146-56. doi: 10.1093/gerona/56.3.M146
13. Dent E, Lien C, Lim WS, et al. The Asia-Pacific clinical practice guidelines for the management of frailty. J Am Med Dir Assoc 2017;18(7):564-75.

14. Puts MTE, Toubasi S, Andrew MK, Ashe MC, Ploeg J, Atkinson E, et al. Interventions to prevent or reduce the level of frailty in communitydwelling older adults: A scoping review of the literature and international policies. Age Ageing 2017;46(3):383-92. doi: 10.1093/ageing/afw247.

15. Zaslavsky O, Thompson H, Demiris G. The role of emerging information technologies in frailty assessment. Res Gerontol Nurs 2012;5(3):216-28. doi: 10.3928/19404921-20120410-02.

16. Lang PO, Michel JP, Zekry D. Frailty syndrome: A transitional state in a dynamic process. Gerontology 2009;55(5):539-49. doi: 10.1159/000211949.

17. World Health Organization. World report on ageing and health. Geneva: WHO, 2015. Available at https://apps.who.int/iris/ bitstream/handle/10665/186463/97892406 94811_eng.pdf;jsessionid=6AEA60271E19E3F 1C3BB07ED357BF159? sequence $=1$ [Accessed 23 May 2019].

18. Woo J. Designing fit for purpose health and social services for ageing populations. Int J Environ Res Public Health 2017;14(5):457. doi: 10.3390/ ijerph14050457.

19. Lim WS, Wong SF, Leong I, Choo P, Pang WS. Forging a frailty-ready healthcare system to meet population ageing. Int J Environ Res Public Health 2017;14(12):E1448. doi: 10.3390/ijerph14121448.

20. Fougère $B$, Sirois $M J$, Carmichael $\mathrm{PH}$, et al. General practitioners' clinical impression in the screening for frailty: Data from the FAP study pilot. JAMDA 2017;18(2):193.e1-193.e5. doi: 10.1016/j. jamda.2016.11.008.

21. Pialoux T, Goyard J, Lesourd B. Screening tools for frailty in primary health care: A systematic review. Geriatr Gerontol Int 2012;12(2):189-97. doi: 10.1111/j.1447-0594.2011.00797.x.

22. Reeves D, Pye S, Ashcroft DM, et al. The challenge of ageing populations and patient frailty: can primary care adapt? BMJ 2018;362(k3349). doi: 10.1136/bmj.k3349.

23. Chapman S, Thomas S. Falls and the rise of the GP contract: An EMIS web protocol and template to help identify frail patients. $\mathrm{Br} J$ Community Nurs 2017;22(11):554-56. doi: 10.12968/ bjen.2017.22.11.554.

24. Lacas A, Rockwood K. Frailty in primary care: A review of its conceptualization and implications for practice. BMC Med 2012;10(4). doi: 10.1186/17417015-10-4.

25. Bock JO, König HH, Brenner $\mathrm{H}$, et al. Associations of frailty with health care costs - results of the ESTHER cohort study. BMC Health Serv Res 2016;16(128):1-11. doi: 10.1186/s12913-016-1360-3.

26. Dent E, Kowal P, Hoogendijk EO. Frailty measurement in research and clinical practice: A review. Eur J Intern Med 2016;31:3-10. doi: 10.1016/j.ejim.2016.03.007.

27. Saleh PY, Maréchal F, Bonnefoy M, Girier $P$ Krolak-Salmon P, Letrilliart L. Views of general practitioners on the frailty in elderly: a qualitative study. Gériatrie Psychol Neuropsychiatr du Vieil 2015;13(3):272-78. doi: 10.1684/pnv.2015.0552.

28. Korenvain C, Famiyeh IM, Dunn S, Whitehead CR, Rochon PA, McCarthy LM. Identifying frailty in primary care: A qualitative description of family physicians' gestalt impressions of their older adult patients. BMC Fam Pract 2018;19(61):1-7. doi: 10.1186/s12875-018-0743-4. 
29. Bleijenberg N, Ten Dam VH, Steunenberg B, et al. Exploring the expectations, needs and experiences of general practitioners and nurses towards a proactive and structured care programme for frail older patients: A mixed-methods study. J Adv Nurs 2013;69(10):2262-73. doi: 10.1111/jan.12110.

30. Archibald MM, Ambagtsheer R, Beilby J, et al. Perspectives off frailty and frailty screening: Protocol for a collaborative knowledge translation approach and qualitative study of stakeholder understandings and experiences. BMC Geriatr 2017;17(87)1-8. doi: 10.1186/s12877017-0483-7.

31. Asbury JE. Overview of Focus Group Research. Qual Health Res 1995;5(4):414-20. doi: 10.1177/104973239500500402.

32. Webb C, Kevern J. Focus groups as a research method: A critique of some aspects of their use in nursing research. J Adv Nurs 2001;33(6):798-805. doi: 10.1046/j.1365-2648.2001.01720.x

33. Onwuegbuzie AJ, Collins KMT. A typology of mixed methods sampling designs in social science research. Qual Rep 2007;12(2):281-316.

34. Quine S, Bernard D, Booth $M$, et al. Health and access issues among Australian adolescents: A rural-urban comparison. Rural and Remote Health 2003:245:1-11.

35. Smith KB, Humphreys JS, Wilson MG. Addressing the health disadvantage of rural populations: How does epidemiological evidence inform rural health policies and research? Aust J Rural Health 2008;16(2):56-66. doi: 10.1111/j.14401584.2008.00953.x.

36. Thomas SL, Wakerman J, Humphreys JS. Ensuring equity of access to primary health care in rural and remote Australia - What core services should be locally available? Int J Equity Health 2015;14(111):1-8. doi: 10.1186/s12939-015-0228-1.

37. Pong RW, DesMeules M, Lagacé C. Rural-urban disparities in health: How does Canada fare and how does Canada compare with Australia? AJRH 2009;17(1):58-64. doi: 10.1111/j.14401584.2008.01039.x.

38. McAuley RG, Paul WM, Morrison GH, Beckett RF, Goldsmith $\mathrm{CH}$. Five-year results of the peer assessment program of the college of physicians and surgeons of Ontario. CMAJ 1990;143(11):1193-99.

39. Britt H, Valenti L, Miller GC. Time for care: Length of general practice consultations in Australia. Aust Fam Physician 2002;31(9):876-80.

40. Charles J, Britt $H$, Valenti L. The independent effect of age of general practitioner on clinical practice. Med J Aust 2006;185(2):105-09. doi: 10.5694/j.1326-5377.2006.tb00485.x

41. Ritchie J, Spencer L. Qualitative data analysis for applied policy reasearch. In: Huberman, AM, Miles M, editors. Qualitative researcher's companion. Thousand Oaks, CA: SAGE Publications, 2002; p. 305-29.

42. Parkinson S, Eatough V, Holmes J, Stapley E Midgley N. Framework analysis: a worked example of a study exploring young people's experiences of depression. Qual Res Psychol 2016;13(2):109-29. doi: 10.1080/14780887.2015.1119228.

43. Srivastava A, Thomson SB. Framework analysis: A qualitative methodology for applied policy research. J Adm Gov 2009;4(2):72-79.

44. Hamirudin AH, Ghosh A, Charlton $\mathrm{K}$ Bonney A, Walton K. Trends in uptake of the 75+ health assessment in Australia: A decade of evaluation. Aust J Prim Health 2015;21(4)423-28. doi: 10.1071/PY14074.

45. Kaethler Y, Molnar F, Mitchell SL, Soucie P, Man-Son-Hing M. Defining the concept of frailty: A survey of multi-disciplinary health professionals. Geriatr Today. 2003;6:26-31.
46. Bergman H, Ferrucci L, Guralnik J, et al. Frailty: An emerging research and clinical paradigm - Issues and controversies. J Gerontol A Biol Sci Med Sci 2007:62(7):731-37. doi: 10.1093/gerona/62.7.731.

47. Warmoth $K$, Lang IA, Phoenix $C$, et al. 'Thinking you're old and frail': A qualitative study of frailty in older adults. Ageing Soc 2016;36(7):1483-1500. doi: 10.1017/S0144686X1500046X.

48. Fried LP, Ferrucci L, Darer J, Williamson JD, Anderson, G. Untangling the concepts of disability, frailty, and comorbidity: Implications for improved targeting and care. J Gerontol A Biol Sci Med Sci 2004;59(3):255-63. doi: 10.1093/ gerona/59.3.M255.

49. Theou O, Rockwood MRH, Mitnitski A, Rockwood K. Disability and co-morbidity in relation to frailty: How much do they overlap? Arch Gerontol Geriatr 2012;55(2):e1-8. doi: 10.1016/j archger.2012.03.001.

50. Tavassoli N, Guyonnet S, Abellan Van Kan G, et al. Description of 1,108 older patients referred by their physician to the 'Geriatric Frailty Clinic (G.F.C) for assessment of frailty and prevention of disability' at the gerontopole. J Nutr Heal Aging 2014;18(5):457-64.

51. Gwyther H, Shaw R, Jaime Dauden EA, et al. Understanding frailty: A qualitative study of European healthcare policy-makers' approaches to frailty screening and management. BMJ Open 2018;8(1):e018653. doi: 10.1136/bmjopen-2017018653.

52. D’Avanzo B, Shaw R, Riva S, et al. Stakeholders' views and experiences of care and interventions for addressing frailty and pre-frailty: A metasynthesis of qualitative evidence. PLoS One 2017;12(7):e0180127.

53. Sutorius FL, Hoogendijk EO, Prins $B A H$, Van Hout HPJ. Comparison of 10 single and stepped methods to identify frail older persons in primary care: Diagnostic and prognostic accuracy. BMC Fam Pract 2016;17(102). doi: 10.1186/s12875016-0487-y.

54. Hoogendijk EO, Van Der Horst H, Deeg DJH, et al. The identification of frail older adults in primary care: Comparing the accuracy of five simple instruments. Age Ageing 2013;42(2):262-65. doi: 10.1093/ageing/afs163.

55. Clegg A, Young J, Iliffe S, Rikkert MO, Rockwood K. Frailty in elderly people. Lancet 2013;381(9868):752-62. doi: 10.1016/S01406736(12)62167-9.

56. Sacha J, Sacha M, Sobon J, Borysiuk Z, Feusette $P$. Is it time to begin a public campaign concerning frailty and pre-frailty? A review article. Front Physiol 2017;8(484). doi: 10.3389/ fphys.2017.00484 